\title{
When ophthalmologists step up to the COVID-19 frontlines
}

\author{
Kelvin Zhenghao $\mathrm{Li}^{1} \cdot$ Vernon Khet Yau Yong ${ }^{1,2} \cdot$ Llewellyn Kuan Ming Lee ${ }^{1,2} \cdot$ Chee Fang Chin ${ }^{1} \cdot$ \\ Leonard Wei Leon Yip $\mathbb{D}^{1,2}$
}

Received: 1 April 2020 / Revised: 16 April 2020 / Accepted: 20 April 2020 / Published online: 28 April 2020

(C) The Royal College of Ophthalmologists 2020

\section{To the Editor:}

A novel coronavirus was first reported in Wuhan city, China on 31 December 2019, and by 23rd January 2020, Singapore confirmed its first COVID-19 case. In the initial period, Family Physicians and Emergency Physicians served as frontline medical staff in screening and treating COVID-19 cases.

\section{Preparation and planning}

Drawing from experiences from the 2003 severe acute respiratory syndrome (SARS) outbreak in Singapore, the National Centre for Infectious Disease (NCID), a 330-bed capacity national facility was set up to handle infectious disease outbreaks. During such an outbreak, physicians and anaesthetists are critically needed in the outbreak wards and intensive care units. Ophthalmologists can contribute meaningfully during such times too.

Since 31st January 2020, ophthalmologists, together with various surgical disciplines, augmented the staffing levels of $\mathrm{NCID}$, the main centre where COVID-19 suspects were screened and treated. The role was to run the screening centre (SC), where suspect cases were screened. As of 31st March 2020, 51 ophthalmologists of all ranks (Medical Officers to Consultants) had been deployed to the SC safely. Recognising that age and co-morbidities result in less favourable outcomes in COVID-19 patients, those of age $>60$ years old and/or with cardiovascular risk factors [1] were excluded from deployment.

Leonard Wei Leon Yip

leonard_yip@ttsh.com.sg

1 Department of Ophthalmology, Tan Tock Seng Hospital, National Healthcare Group, Singapore, Singapore

2 Lee Kong Chian School of Medicine, National Technological University, Singapore, Singapore
Admittedly, the average ophthalmologist is ill-prepared to step up to the COVID-19 frontline after years of not using a stethoscope, reading chest radiographs, using personal protective equipment (PPE) and performing diagnostic swabs.

At NCID, our preparation consisted of 'Just-in-time' training conducted by emergency physicians with inputs from infectious disease colleagues. Since COVID-19 is predominantly transmitted via respiratory droplets and contact routes, mask fitting exercises were conducted for all staff and usage of PPE were both taught in-person and online.

\section{Deployment}

In the SC, patients were clerked and examined by ophthalmologists in full PPE (hair cover, eye shields or goggles, N95 masks, gowns and gloves). Emphasis was placed on patients with significant travel or contact history. Fortunately, subsequent investigations and managements followed a strict management protocol, which was constantly updated. Chest radiographs were used to detect lung changes consistent with COVID-19 [2]. These were expeditiously reported by a radiologist throughout the day. Majority of those screened received nasopharyngeal diagnostic swabs, which were performed by trained nurses.

Reflecting on the ongoing experience, we realise we are fortunate to serve in a structured and well-resourced setup. However, we are cognizant that the journal reader's local context may differ. It is a responsibility to upskill to face developing challenges.

We leave the reader with resources related to COVID-19 for departmental or self-directed learning. These encompass understanding COVID-19 clinical characteristics [1], use of full PPE [3], chest radiograph interpretation [2], procedural steps for nasopharyngeal swabs [4] and an algorithm for the acute management of COVID-19 [5]. It is our hope that these resources enhance the ophthalmologist's ability to perform in this unprecedented setting. 


\section{Conclusion}

As the COVID-19 pandemic spreads worldwide, healthcare workers around the world have a critical role in the fight against COVID-19. Despite being a niche specialty, ophthalmologists can also contribute significantly at the frontlines.

Acknowledgements We acknowledge all doctors, nurses and allied health at NCID.

Funding The authors received no financial support with respect to the research, authorship and/or publication of this article.

\section{Compliance with ethical standards}

Conflict of interest The authors declare no conflict of interest.

Publisher's note Springer Nature remains neutral with regard to jurisdictional claims in published maps and institutional affiliations.

\section{References}

1. Wang $\mathrm{D}, \mathrm{Hu} \mathrm{B}, \mathrm{Hu} \mathrm{C}$, Zhu F, Liu X, Zhang $\mathrm{J}$ et al. Clinical characteristics of 138 hospitalized patients with 2019 novel coronavirus-infected pneumonia in Wuhan, China. JAMA. 2020.

2. Wong HY, Lam HY, Fong AH, Leung ST, Chin TW, Lo CS et al. Frequency and distribution of chest radiographic findings in COVID-19 positive patients. Radiology. 2020.

3. Health Protection Scotland. COVID-19 - the correct order for donning, doffing and disposal of Personal Protective Equipment (PPE) for healthcare workers (HCWs) in a secondary care setting. Available from: https://www.hps.scot.nhs. uk/web-resources-container/covid-19-the-correct-order-for-donningdoffing-and-disposal-of-personal-protective-equipment-ppe-for-hea 1thcare-workers-hows-in-a-secondary-care-setting/ [Accessed 1 April 2020].

4. Centers for disease control and prevention. Influenza Specimen Collection. Available from: https://www.cdc.gov/flu/pdf/freeresources/healthcare/flu-specimen-collection-guide.pdf [Accessed 1 April 2020].

5. Zhang J, Zhou L, Yang Y, Peng W, Wang W, Chen X. Therapeutic and triage strategies for 2019 novel coronavirus disease in fever clinics. Lancet Respiratory Med 2020;8:e11-2. 